\title{
Fluxonium Steps up to the Plate
}

\section{A decade-old alternative to the leading superconducting qubit exhibits the coherence times needed for applications.}

\section{by Gianluigi Catelani*}

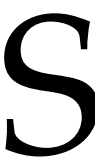
uperconducting qubits are in the news thanks to Google. When researchers at the company claimed in October to have achieved "quantum supremacy" over classical computers, they based their assertion on a circuit of 53 qubits made with superconducting elements. Each of these qubits is known as a transmon-a tiny electrical circuit with discrete energy levels like those of an atom [1]. But transmons are not the only game in town, and alternative types of "artificial atoms" may be preferable for certain quantum technologies. New experiments from Vladimir Manucharyan and colleagues at the University of Maryland, College Park, may rekindle interest in a superconducting qubit introduced ten years ago, the fluxonium [2]. This qubit's features are attractive for building circuits with large numbers of qubits. But the short lifetime of its quantum states-or "coherence time" - has meant that it could handle only a few successive operations (gates). The Maryland team has now demonstrated fluxonium qubits with coherence times that are roughly 10 times longer than previous realizations, enough to make the qubits a serious contender for the next generation of quantum circuits [3].

The first superconducting version of an artificial atom was the Cooper pair box [4]. Celebrating its 20th anniversary this year, this simple circuit (Fig. 1, left) consists of a

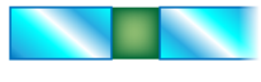

Cooper pair box

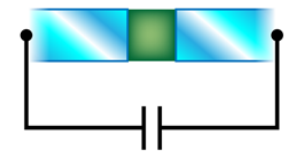

Transmon

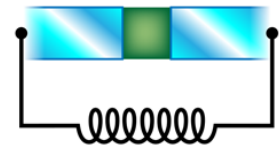

Fluxonium
Figure 1: Simplified representations of the circuitry in three types of superconducting qubits. All three qubits share the same central element, a Josephson junction formed by a thin insulating barrier (green) separating two superconducting electrodes (blue). The electrodes are shunted by a capacitor in the transmon and by an inductor in the fluxonium. (APS/Alan Stonebraker)

\footnotetext{
*JARA Institute for Quantum Information (PGI-11), Forschungszen-
} trum Juelich $\mathrm{GmbH}$, Juelich, Germany small superconducting island (the box) and a larger "reservoir" of superconducting electron pairs (Cooper pairs). A nanometer-scale insulating barrier separates the island and the reservoir, forming a Josephson junction. This junction ensures that the circuit's energy levels are unequally spaced (anharmonic), such that two of the energy levels are far from other levels and can serve as the qubit states.

The higher a qubit's anharmonicity, the faster it can complete an operation of a given accuracy, so it may seem that enhancing anharmonicity is the way to go. The transmon, however, has a much lower anharmonicity than the Cooper pair box because its Josephson junctions are shunted by a capacitor (Fig. 1, center). The capacitor suppresses the device's sensitivity to charge noise, endowing the transmon with a long coherence time [5]. This increase in coherence time more than makes up for the slowing down of the gates, enabling more quantum operations to be performed successfully. But clearly, packing a long coherence time and large anharmonicity into one qubit would be ideal, which is why researchers are investigating alternatives.

Fluxonium offered this potential combination when it came on the scene in 2009. The shunting element for this qubit is an inductor (Fig. 1, right), which suppresses chargenoise sensitivity while preserving large anharmonicity. The inductive loop allows the qubit frequency $f$ (the energy difference between the qubit's two quantum states) to be tuned via a magnetic field. This tuning is useful when designing gates because it allows the energies of two qubits to be aligned, enhancing their interaction and speeding up operations. But it makes the qubit more sensitive to flux noise, which can severely limit coherence. To reduce this sensitivity, researchers therefore typically operate the device at a "sweet spot," where half of a magnetic-flux quantum goes through the inductive loop, and the qubit frequency is at its minimum.

In absolute numbers, fluxonium's best coherence time to date $(\sim 10 \mu \mathrm{s})$ [7] is comparable to that of the transmons used by Google (16 $\mu$ s) (see note in Ref. [6]). But a better figure of merit for qubits is their quality factor (the product of the coherence time and $2 \pi f$ ), which allows a comparison of qubits that operate at different frequencies. The Google experiment used 7-GHz qubits, corresponding to a quality factor of $7 \times 10^{5}$. (State-of-the-art transmons have quality factors that are 10 times bigger but only in circuits with a few qubits.) Fluxonium qubits operate at lower frequency 


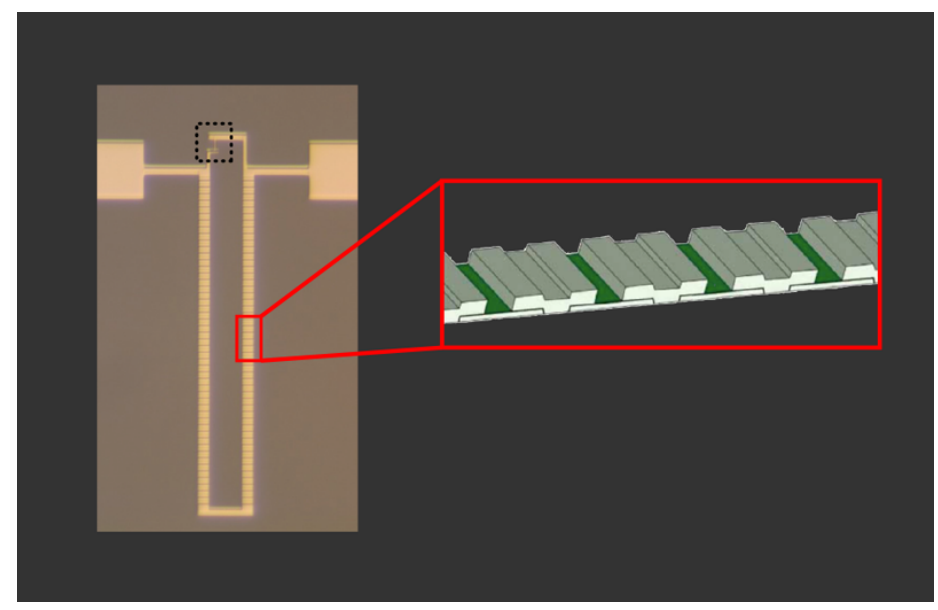

Figure 2: One of the fluxonium devices fabricated by Manucharyan and colleagues. The dotted square at upper left shows the central Josephson junction. The shunting inductor is made up of an array of Josephson junctions (detail shown at right.) (L. B. Nguyen et al. [3])

(under $1 \mathrm{GHz}$ ), meaning a quality factor below $10^{5}$. The University of Maryland team reports coherence times of around $100 \mu \mathrm{s}$ when their device is operated at the sweet spot, a factor of 10 improvement that brings the quality factor close to that of Google's transmons.

The shunting inductor in their fluxonium devices (Fig. 2) is more complicated than a coiled metallic wire, which would provide too low of an inductance [2]. Instead, the team exploits a superconductor's so-called kinetic inductance. By definition, an inductor opposes a change in the current flowing through it. In a superconductor, however, this opposition originates from the inertia of the material's moving Cooper pairs, rather than the geometry of the wire. As in previous studies [2,7], the Maryland team maximizes this kinetic inductance by fabricating a "superinductor" —an array of several tens to about a hundred Josephson junctions.

It is not clear what led to the improved coherence times, since the current design is not that different from previous ones. One possible explanation is that the researchers have combined the junction fabrication technique of Ref. [2] with the 3D cavity approach of Ref. [7]. The latter, which was pioneered for transmons [8], has the advantage that the cavity protects the circuit from unwanted electromagnetic noise, while allowing manipulation and readout of the qubit. However, the superconducting electrodes that couple the qubit to the cavity add some capacitance, which makes the qubit interact with defects in the substrate or in the various materials making up the device. These defects can absorb energy from the qubit and, according to the researchers, may be the main driver of decoherence, as is the case for most transmons.

Despite these uncertainties, the new work serves as a proof-of-principle that fluxonium is a viable alternative to the transmon. The next step is perfecting the device. It should be noted that the substrate material and fabrication process used were not at the state-of-the-art level, so the authors plausibly argue that even longer coherence times are within reach. Also, an important lesson from this work and others [8] is that incorporating junction arrays of various sizes doesn't cause significant decoherence. This realization bodes well for investigating more complicated circuits such as that considered in Ref. [9]. Alternatively, superconducting wires could be used instead of junction arrays for the superinductor, a possibility that was explored in two experiments earlier this year $[10,11]$.

The next important step is to demonstrate fast and accurate gates with coupled fluxonium qubits. The Maryland team is working toward this feat already. For example, to take advantage of the flux control of their fluxonium qubits means moving away from the sweet spot. They have found this change reduces the coherence time to about $5 \mu$ s because of flux noise. But the reduced coherence time is still long compared to the typical gate time ( $100 \mathrm{~ns})$, making fluxcontrolled gates a possibility.

This research is published in Physical Review X.

\section{REFERENCES}

[1] F. Arute et al., "Quantum supremacy using a programmable superconducting processor," Nature 574, , 505 (2019).

[2] V. E. Manucharyan, J. Koch, L. I. Glazman, and M. H. Devoret, "Fluxonium: Single Cooper-pair circuit free of charge offsets," Science 326, 113 (2009).

[3] L. B. Nguyen, Y.-H. Lin, A. Somoroff, R. Mencia, N. Grabon, and V. E. Manucharyan, "High-coherence fluxonium qubit," Phys. Rev. X 9, 041041 (2019).

[4] Y. Nakamura, Yu. A. Pashkin, and J. S. Tsai, "Coherent control of macroscopic quantum states in a single-Cooper-pair box," Nature 398, 786 (1999).

[5] J. Koch, T. M. Yu, J. Gambetta, A. A. Houck, D. I. Schuster, J. Majer, A. Blais, M. H. Devoret, S. M. Girvin, and R. J. Schoelkopf, "Charge-insensitive qubit design derived from the Cooper pair box," Phys. Rev. A 76, No. 4 (2007).

[6] The quoted coherence time is technically the device's energy relaxation time, but in today's transmons these numbers are usually close.

[7] I. M. Pop, K. Geerlings, G. Catelani, R. J. Schoelkopf, L. I. Glazman, and M. H. Devoret, "Coherent suppression of electromagnetic dissipation due to superconducting quasiparticles," Nature 508, 369 (2014).

[8] H. Paik et al., "Observation of high coherence in Josephson junction qubits measured in a three-dimensional circuit QED architecture," Phys. Rev. Lett. 107, 240501 (2011).

[9] S. Richer, N. Maleeva, S. T. Skacel, I. M. Pop, and D. DiVincenzo, "Inductively shunted transmon qubit with tunable transverse and longitudinal coupling," Phys. Rev. B 96, 174520 (2017). 
[10] T. M. Hazard, A. Gyenis, A. Di Paolo, A. T. Asfaw, S. A. Lyon, A. Blais, and A. A. Houck, "Nanowire superinductance fluxonium qubit," Phys. Rev. Lett. 122, 010504 (2019).

[11] L. Grünhaupt et al., "Granular aluminium as a superconducting material for high-impedance quantum circuits," Nat. Mater. 18, 816 (2019).

10.1103/Physics.12.131 